\title{
A shadow of the repulsive Rutherford scattering and Hamilton vector
}

\author{
D A Shatilov ${ }^{1}$ and Z K Silagadze ${ }^{1,2}$ \\ ${ }^{1}$ Novosibirsk State University, Novosibirsk 630090, Russia \\ E-mail: d.shatilov@g.nsu.ru \\ ${ }^{2}$ Budker Institute of Nuclear Physics, Novosibirsk 630 090, Russia \\ E-mail: Z.K.Silagadze@inp.nsk.su
}

\begin{abstract}
The fact that repulsive Rutherford scattering casts a paraboloidal shadow is rarely exploited in introductory mechanics textbooks. Another rarely used construction in such textbooks is the Hamilton vector, a cousin of the more famous LaplaceRunge-Lenz vector. We will show how the latter (Hamilton's vector) can be used to explain and clarify the former (paraboloidal shadow).
\end{abstract}




\section{Introduction}

Recently, in an interesting paper [1, a shadow of the repulsive Rutherford scattering was considered in great detail in fixed-target and center-of-mass frames. It was noted that this shadow, in addition to playing an important role in low-energy ion scattering spectroscopy, is of great educational value.

Despite its educational attractiveness, this simple fact concerning repulsive Coulomb orbits was seemingly forgotten. After the boundary parabola equation was derived for the envelope of a family of scattered orbits in 2, the problem did not attract attention and disappeared into oblivion, as witnessed by the accidental rediscovery of parabolic shadow by an undergraduate student in computer simulations [3].

In this article, we will show how the Hamilton vector can be used to obtain the paraboloidal shape of the shadow in a simple and transparent way. We hope that the publication of [1] will stimulate interest in this neither too trivial nor too difficult problem, and help it to finally find its way into introductory textbooks on classical mechanics.

\section{Hamilton vector}

The Hamilton vector is an extra constant of motion in the Kepler problem, like the well-known Laplace-Runge-Lenz vector (in fact, the two are simply related) [4]. This "lost sparkling diamond of introductory level mechanics" [5] was well known in the past, but then almost forgotten (see [4, 6, 7, 8] and references therein).

A great virtue of the Hamilton vector is that it can be introduced in a simple and natural way 9 . Let

$$
\mathbf{e}_{r}=\cos \phi \mathbf{i}+\sin \phi \mathbf{j}, \quad \mathbf{e}_{\phi}=-\sin \phi \mathbf{i}+\cos \phi \mathbf{j},
$$

be time-dependent polar-system unit vectors. Then (the dot denotes time-derivative)

$$
\dot{\mathbf{e}}_{r}=\dot{\phi} \mathbf{e}_{\phi}, \quad \dot{\mathbf{e}}_{\phi}=-\dot{\phi} \mathbf{e}_{r} .
$$

Using the second equation in (2), we can write Newton's second law for the Coulomb/Kepler problem in the form (in fixed-target frame)

$$
\dot{\mathbf{V}}=\frac{\alpha}{\mu r^{2}} \dot{\mathbf{e}}_{r}=-\frac{\alpha}{\mu r^{2} \dot{\phi}} \dot{\mathbf{e}}_{\phi}=\frac{\mathrm{d}}{\mathrm{d} t}\left(-\frac{\alpha}{L_{z}} \mathbf{e}_{\phi}\right),
$$

where $\alpha=\frac{Z_{p} Z_{t} e^{2}}{4 \pi \epsilon_{0}}, Z_{p}$ and $Z_{t}$ being the projectile and target charges, respectively, in units of the elementary charge $e$ and $\mathbf{V}$ is the the target-relative projectile velocity. At

the last step we have used that $L_{z}=\mu r^{2} \dot{\phi}, z$-component of the total angular momentum of the system, is conserved in the central field (like any other components of the angular momentum). It is important to note that, similar to the traditional formulation of the two-body problem, a reduced mass of the system is introduced

$$
\mu=\frac{m_{p} m_{t}}{m_{p}+m_{t}}
$$


where $m_{p}$ and $m_{t}$ are the projectile and target masses in the specified order. Equation (3) indicates that the following vector (the Hamilton vector) is conserved in the Coulomb/Kepler field:

$$
\mathbf{H}=\mathbf{V}+\frac{\alpha}{L_{z}} \mathbf{e}_{\phi} .
$$

The physical meaning of the Hamilton vector is especially clear in the case of an attractive potential, when $\alpha<0$. Then

$$
\mathbf{H}=\mathbf{V}-\mathbf{V}_{\mathbf{c}},
$$

where $\mathbf{V}_{\mathbf{c}}=\frac{|\alpha|}{L_{z}} \mathbf{e}_{\phi}$ is the velocity that the particle would have if it moved in a circular orbit around the center of the field with the same $L_{z}$ component of the angular momentum. Therefore, the Hamilton vector is a kind of velocity defect (deficit) that is conserved during motion in the Coulomb/Kepler field.

Note that the better known Laplace-Runge-Lenz vector $\mathbf{A}$ is related to the Hamilton vector:

$$
\mathbf{A}=\mathbf{H} \times \mathbf{L}=\mathbf{V} \times \mathbf{L}+\alpha \mathbf{e}_{r}
$$

\section{The shape of the shadow}

Let us consider some projectile trajectory (in the fixed-target frame) launched at $x=-\infty$ and with an initial velocity $\mathbf{V}_{\infty}$ directed along the $\mathrm{x}$ axis. Selected point $B$ at this trajectory has the polar angle $\phi$. When point $A$ on this trajectory moves towards negative infinity on the $x$-axis, the corresponding unit vector $\mathbf{e}_{\phi}$ approaches negative direction of the $y$ axis, and its $x$-projection tends to zero (see figure 1). Let us

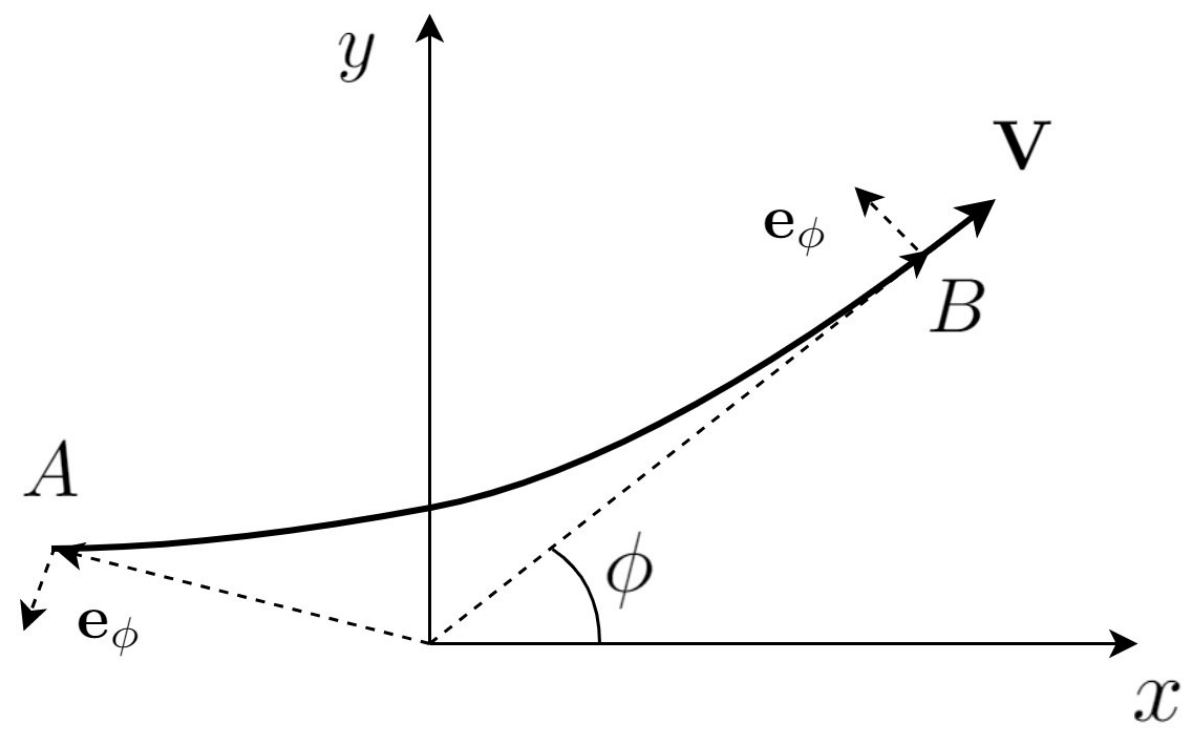

Figure 1. Projectile trajectory along which $\mathbf{H}(A)=\mathbf{H}(B)$ for any choice of $A$ and $B$. 
write the conservation law of the Hamilton vector in the $x$ and $y$-projections for points $A$ and $B$ in the limit, when the point $A$ corresponds to $x=-\infty$ :

$$
V_{\infty}=V_{x}-\frac{\alpha}{L_{z}} \sin \phi,-\frac{\alpha}{L_{z}}=V_{y}+\frac{\alpha}{L_{z}} \cos \phi
$$

where $\mathbf{V}=\left(V_{x}, V_{y}\right)$ is the projectile velocity at point $B$. Then

$$
\begin{aligned}
& V^{2}=\left(V_{\infty}+\frac{\alpha}{L_{z}} \sin \phi\right)^{2}+\frac{\alpha^{2}}{L_{z}^{2}}(1+\cos \phi)^{2}= \\
& V_{\infty}^{2}+2 \frac{\alpha^{2}}{L_{z}^{2}}+2 \frac{\alpha}{L_{z}} V_{\infty} \sin \phi+2 \frac{\alpha^{2}}{L_{z}^{2}} \cos \phi .
\end{aligned}
$$

On the other hand, $V^{2}$ can be determined from the energy conservation law

$$
\frac{\mu V_{\infty}^{2}}{2}=\frac{\mu V^{2}}{2}+\frac{\alpha}{r}
$$

which gives

$$
V^{2}=V_{\infty}^{2}-2 \frac{\alpha}{\mu r}
$$

where $r$ is the target-relative distance at point $B$. Then, substituting (11) into the l.h.s. of (9), we get after a simple algebra

$$
\frac{1}{\mu r}=-\alpha \sigma^{2}(1+\cos \phi)+\sigma V_{\infty} \sin \phi
$$

where $\sigma=\frac{1}{\left|L_{z}\right|}$ (note that $L_{z}<0$, because $\dot{\phi}<0$ ). This formula describes every possible (for different initial parameters) projectile trajectory, which is a hyperbola in polar coordinates.

The rationale in finding shadow shape from this point can be followed as in [1]. Namely, for a given polar angle $\phi$, the shadow boundary is determined by the minimum value of $r$. Therefore, we must choose $\sigma_{m}$ (inverse angular momentum) in such a way that

$$
\left.\frac{\mathrm{d}}{\mathrm{d} \sigma}\left[-\alpha \sigma^{2}(1+\cos \phi)+\sigma V_{\infty} \sin \phi\right]\right|_{\sigma=\sigma_{m}}=0 .
$$

This gives

$$
\sigma_{m}=\frac{V_{\infty} \sin \phi}{2 \alpha(1+\cos \phi)}
$$

To calculate the minimum value of $r$ for a given $\phi$, we substitute (14) into (12) and get

$$
\frac{1}{\mu r_{\text {min }}}=\frac{V_{\infty}^{2} \sin ^{2} \phi}{4 \alpha(1+\cos \phi)}=\frac{V_{\infty}^{2}}{4 \alpha}(1-\cos \phi) .
$$

Therefore, the boundary of the shadow is given by the equation

$$
\frac{1}{r_{\min }}=\frac{\mu V_{\infty}^{2}}{4 \alpha}(1-\cos \phi)
$$

which is the equation of the parabola in polar coordinates.

Note that finding the envelope of trajectories from the equation 12 can be put in a general envelope determination context by treating this formula as a condition 
$F(r, \phi, \sigma)=0$ and then applying the general rule for the envelope $\frac{\partial}{\partial \sigma} F(r, \phi, \sigma)=0$ (more on this in the concluding remarks).

So far, we have implicitly assumed that all possible projectile trajectories are confined to the $x-y$ plane. In the case of three-dimensional flow, due to the axial symmetry of the problem, the shape of the shadow is a paraboloid obtained by rotating the parabola (16) around its axis of symmetry.

Introducing the cylindrical coordinates $z=r_{\min } \cos \phi, \rho=r_{\min } \sin \phi$, equation (16) can be rewritten in the form

$$
\frac{4 \alpha}{\mu V_{\infty}^{2}}=\sqrt{\rho^{2}+z^{2}}-z .
$$

Solving for $z$, we obtain the following equation for the shadow paraboloid in cylindrical coordinates:

$$
z=\frac{\rho^{2}}{8 \xi}-2 \xi
$$

where

$$
\xi=\frac{\alpha}{m V_{\infty}^{2}}
$$

\section{Concluding remarks}

As we can see, using the Hamilton vector allows a simple and transparent way to get the main result of [1], the equation (18) for the shape of the shadow. In fact, we have been using this problem for some time as an exercise in an introductory mechanics course at Novosibirsk State University [10, 11]. In [11], two more solutions to the problem are given.

The first solution is based on the fact that the shadow boundary is the envelope of the projectile trajectories. A one-parameter family of the projectile trajectories is given by the equation (for details, see [11]. Note that in [11] the flow falls from the right).

$$
F\left(r, \phi, \phi_{0}\right)=\frac{\alpha}{\mu V_{\infty}^{2}} \frac{\tan ^{2} \phi_{0}}{r}+1+\frac{\cos \left(\phi-\phi_{0}\right)}{\cos \phi_{0}}=0,
$$

where $\phi_{0}$ is the polar angle of the symmetry axis of the hyperbolic trajectory. Then it is known from mathematics (for inquisitive readers: there are some subtleties in the mathematical definition of an envelope, see [12, 13, 14]) that the envelope equation is obtained by excluding the parameter $\phi_{0}$ from the system

$$
\begin{aligned}
& F\left(r, \phi, \phi_{0}\right)=0, \\
& \frac{\partial}{\partial \phi_{0}} F\left(r, \phi, \phi_{0}\right)=0 .
\end{aligned}
$$

In this way we can obtain the envelope equation

$$
\frac{1}{r_{\min }}=\frac{\mu V_{\infty}^{2}}{4 \alpha}(1-\cos \phi)
$$


The sign in front of $\cos \phi$ can change assuming that the flow falls from the right, for example, as it is done in [11]. In the present article (as in [1]) it was assumed that the flow falls from the left.

In the second solution method, considered in [11], we fix the polar angle $\phi$ in the family (20) and choose the parameter $\phi_{0}$ so that the corresponding trajectory minimizes $r$. This is the same idea that was used in [1] (and in this article), just the parameterizations in the one-parameter family of projectile trajectories are different. Therefore, we emphasize that the most original contribution relative to [1] is not so much about the procedure for obtaining the shadow itself, but rather obtaining trajectories, which is facilitated by the use of the Hamilton vector.

The method used in this article can be extended to the interesting case when the infalling flux is originated from an emitter at a finite distance from the repulsive Coulomb potential centre. Such a modification of the problem of the shape of the shadow in repulsive Rutherford scattering is also of considerable pedagogical interest. It represents a physical situation that actually occurs in atomic physics, and allows students to become familiar with such interesting phenomena as the glory and rainbow effects in scattering processes [15].

Consider projectile trajectories all starting from some point $A$ on the $x$-axis at a distance $R$ from the center of the field (see figure 2). We assume that the particles are released from the emitter with the same in magnitude initial velocity $V_{0}$. If the initial

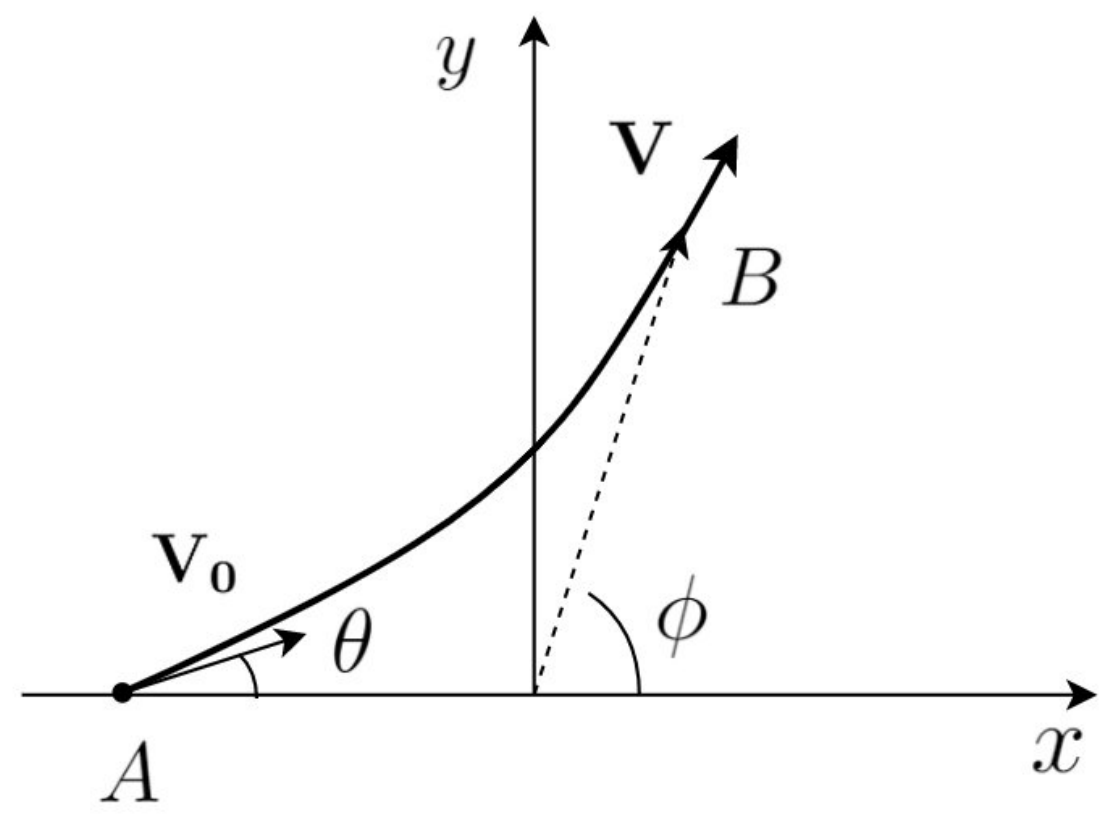

Figure 2. The definition of kinematic variables for the projectile trajectory launched from the point $A$ on the $x$-axis.

velocity $\mathbf{V}_{0}$ makes an angle $\theta$ with the $x$-axis, the conservation equations (8) of the Hamilton vector components will be modified in the following way:

$$
V_{0} \cos \theta=V_{x}-\frac{\alpha}{L_{z}} \sin \phi, \quad V_{0} \sin \theta-\frac{\alpha}{L_{z}}=V_{y}+\frac{\alpha}{L_{z}} \cos \phi .
$$


Then

$$
V^{2}=V_{0}^{2}+2 \frac{\alpha}{L_{z}} V_{0}[\sin (\phi-\theta)-\sin \theta]+2\left(\frac{\alpha}{L_{z}}\right)^{2}(1+\cos \phi),
$$

and using the energy conservation law

$$
\frac{\mu V_{0}^{2}}{2}+\frac{\alpha}{R}=\frac{\mu V^{2}}{2}+\frac{\alpha}{r}
$$

we derive the following relation

$$
\begin{aligned}
& \frac{1}{\mu r}=-\alpha \sigma^{2}(1+\cos \phi)+\sigma V_{0} \sin (\phi-\theta)= \\
& -\alpha \sigma^{2}(1+\cos \phi)+V_{0} \sin \phi \sqrt{\sigma^{2}-\frac{1}{\mu^{2} R^{2} V_{0}^{2}}}-\frac{\cos \phi}{\mu R}
\end{aligned}
$$

where we have taken into account that $\frac{1}{\sigma}=\left|L_{z}\right|=\mu V_{0} R \sin \theta$.

The equation (26) indicates that the minimum $r$ for a given $\phi$ corresponds to $\sigma_{m}$ such that

$$
\sqrt{\sigma_{m}^{2}-\frac{1}{\mu^{2} R^{2} V_{0}^{2}}}=\frac{V_{0} \sin \phi}{2 \alpha(1+\cos \phi)}
$$

Substituting $\sigma_{m}$ from (27) into (26), we finally obtain for the shadow boundary the expression

$$
\frac{1}{r_{\min }}=\frac{\mu V_{\infty}^{2}}{4 \alpha}(1-A)\left(1-\frac{1+A}{1-A} \cos \phi\right)
$$

where we have introduced a convenient dimensionless parameter [15, 16]

$$
A=\frac{V_{\infty}^{2}}{V_{0}^{2}}-1=\frac{2 \alpha}{\mu V_{0}^{2} R}
$$

Since $A>0$ equation (28) corresponds to a hyperbola with vertex at the emission point and focus at the force center [15]. As the emission point moves towards infinity, $A \rightarrow 0$ and the hyperbola $(28)$ degenerates into the parabola $(16)$.

For an attractive potential, the envelope of Kepler ellipses, all starting from the same point in space, can be obtained in a similar way [16].

\section{Acknowledgments}

The work is supported by the Ministry of Education and Science of the Russian Federation.

\section{References}

[1] Žugec P and Topić I 2020 A shadow of the repulsive Rutherford scattering in the fixed-target and the center-of-mass frame Eur. J. Phys. 41065005

[2] Adolph J W, Leon Garcia A, Harter W G, McLaughlin G C, Shiffman R R and Surkus V G 1972 Some Geometrical Aspects of Classical Coulomb Scattering Am. J. Phys. 40 1852-1857

[3] Warner R E and Huttar L A 1991 The parabolic shadow of a Coulomb scatterer Am. J. Phys. 59 $755-756$ 
[4] Martínez-y-Romero R P, Núñezez-Yépez H N and Salas-Brito A L 1993 The Hamilton vector as an extra constant of motion in the Kepler problem Eur. J. Phys. 14 71-73

[5] Chashchina O I and Silagadze Z K 2008 Remark on orbital precession due to central-force perturbations Phys. Rev. D 77107502

[6] Munoz G 2003 Vector constants of the motion and orbits in the Coulomb/Kepler problem Am. J. Phys. 71 1292-1293

[7] Wheeler J T 2005 Not so classical mechanics - unexpected symmetries of classical motion Can. J. Phys. 83 91-138

[8] Goldstein H 1976 More on the prehistory of the Laplace or Runge-Lenz vector Am. J. Phys. 44 $1123-1124$

[9] González-Villanueva A, Núñezez-Yépez H N and Salas-Brito A L 1998 The Coulomb problem and Rutherford scattering using the Hamilton vector Rev. Mex. Fis. 44 183-189

[10] Belchenko Yu I, Gilev E A and Silagadze Z K 2008 Problems in mechanics of particles and bodies (Izhevsk: R\&C Dynamics) (in Russian), the first part is reproduced in arXiv:1004.4504

[11] Silagadze Z K 2017 Mechanics and special relativity: tutorial problems with solutions (Novisibirsk: IPC NGU) (in Russian) arXiv:1711.01670

[12] Bruce J W and Giblin P J 1981 What Is an Envelope? Math. Gazette 65 186-192

[13] Bickel K, Gorkin P and Tran T 2020 Applications of envelopes Complex Anal. Synerg. 62

[14] Kalman D 2007 Solving the Ladder Problem on the Back of an Envelope Math. Gazette 80 163-182

[15] Samengo I and Barrachina R O 1994 Rainbow and glory scattering in Coulomb trajectories starting from a point in space Eur. J. Phys. 15 300-308

[16] Laporte O 1970 On Kepler Ellipses Starting from a Point in Space Am. J. Phys. 38 837-840 